\title{
Reliability and Validity of the Korean Version of the Cornell Scale for Depression in Dementia
}

\author{
Hyun Kook Lim ${ }^{1,4}$, Seung Chul Hong', Wang Youn Won², Changtae Hahn ${ }^{3}$ and Chang Uk Lee ${ }^{3,4} \bowtie$ \\ ${ }^{1}$ Department of Psychiatry, St. Vincent Hospital, College of Medicine, The Catholic University of Korea, Suwon, Republic of Korea \\ ${ }^{2}$ Department of Psychiatry, The Saint Paul Hospital, College of Medicine, The Catholic University of Korea, Seoul, Republic of Korea \\ ${ }^{3}$ Department of Psychiatry, Seoul St. Mary's Hospital, College of Medicine, The Catholic University of Korea, Seoul, Republic of Korea \\ ${ }^{4}$ Catholic Argo-Medical Center, The Catholic University of Korea, Seoul, Republic of Korea
}

Objective The aim of this study was to explore the reliability and validity of the Korean version of the Cornell Scale for Depression in Dementia (CSDD-K), a scale for assessment of depression in dementia.

Methods The original CSDD was translated into Korean and the content was verified through back-translation procedures. This study included 59 depressive patients with Alzheimer's disease (AD), 62 non-depressive patients with AD and 36 healthy elderly controls. The subjects were assessed using CSDD-K, the 17-item Hamilton Depression Rating Scale (HAM- $\left.\mathrm{D}_{17}\right)$, the 15-item Korean version of Geriatric Depression Scale $\left(\mathrm{GDS}_{15}\right.$ ) and the Korean version of Mini-mental Status Examination (MMSE-K).

Results In the reliability test, Cronbach's a coefficient and test-retest reliabilities were 0.92 and 0.91 , respectively, indicating that the CS$\mathrm{DD}-\mathrm{K}$ has good internal consistency. There were significant differences in CSDD-K total scores between AD patients with depression and $\mathrm{AD}$ patients without depression $(\mathrm{p}<0.001)$. In the analysis of the concurrent validity of the CSDD-K, there were significant correlations between the CSDD-K and HAM- $\mathrm{D}_{17}(\mathrm{r}=0.91, \mathrm{p}<0.001)$ and between the CSDD-K and $\mathrm{GDS}_{15}(\mathrm{r}=0.75, \mathrm{p}<0.001)$. ROC curve analysis identified a cut-off score of 7 for the CSDD-K, where the sensitivity was $87.5 \%$ and the specificity was $100 \%$. Factor analysis resulted in a fourfactor solution accounting for $63.8 \%$ of the common variance.

Conclusion The CSDD-K showed good reliability and validity for the assessment of depressive symptom severity in $\mathrm{AD}$ patients. The $\mathrm{CSDD}-\mathrm{K}$ is a useful instrument for assessing $\mathrm{AD}$ patients with depressive symptoms in Korean ethnic population.

Psychiatry Investig 2012;9:332-338

Key Words Dementia, Depression, CSDD-K, Reliability, Validity.

\section{INTRODUCTION}

Depressive symptoms, such as depressed mood, loss of volition and low energy, frequently accompany Alzheimer's disease $(\mathrm{AD})$. The prevalence of major depression in $\mathrm{AD}$ patients has been estimated to be $20-25 \% .{ }^{1}$ Depression in $\mathrm{AD}$ patients has been associated with earlier admission into a nursing home, greater impairments in the quality of life, as well as with increased caregiver distress and burden. ${ }^{2,3}$

Over the past years, there has been growing interest in de-

Received: July 4, 2012 Revised: July 23, 2012

Accepted: August 1, 2012 Available online: November 12, 2012

$\triangle$ Correspondence: Chang Uk Lee, MD, PhD

Department of Psychiatry, Seoul St. Mary's Hospital, The Catholic University of Korea College of Medicine, 505 Banpo-dong, Seocho-gu, Seoul 137-701, Republic of Korea

Tel: +82-2-2258-6082, Fax: +82-2-594-3870, E-mail: jihan@catholic.ac.kr

(c) This is an Open Access article distributed under the terms of the Creative Commons Attribution Non-Commercial License (http://creativecommons.org/licenses/by$\mathrm{nc} / 3.0$ ) which permits unrestricted non-commercial use, distribution, and reproduction in any medium, provided the original work is properly cited. veloping tools for assessing depressive symptoms in dementia patients. Several global scales have been developed to evaluate various neuropsychiatric symptoms of dementia. These include the Behavioral Rating Scales for Dementia of the Consortium to Establish a Registry for Alzheimer's Disease Behavior and the Neuropsychiatric Inventory (NPI)., Although self-report instruments such as the Geriatric Depression Scale (GDS), the Zung Scale, and the Beck Depression Inventory have been used to assess depression in dementia, there is controversy about the reliability and validity of self-report instruments in dementia, with some arguing that their utility is diminished in patients with dementia. ${ }^{6}$ The popular Montgomery-Asberg Depression Rating Scale and the Hamilton Depression Rating Scale (HAMD), which are clinician-rated scales, have also been used in assessing depression in dementia. However, as these scales are based on the clinical interview with the patients themselves, there is potential danger of ignorance of the symptoms, which cognitively impaired patients may 
not express properly. To overcome the aforementioned problems, Alexopoulos et al. developed the Cornell Scale for Depression in Dementia (CSDD), which is based on an interview with the patients and the observations of their caregivers. ${ }^{7}$ The CSDD is composed of 19 item measures used to assess the frequency of five symptoms (seven intrusion items and eight avoidance items) associated with depression in dementia. The CSDD evaluates the frequency of symptoms within the previous week on a 4-point scale (a, 0, 1, and 2). Turkish, Japanese, Chinese Brazilian and Danish versions of the CSDD have been shown to have good psychometric properties. ${ }^{8-12}$

The objective of this study was to validate the Korean version of the CSDD in two phases: translation and adaptation of the scale into a clinical language, followed by the determination of inter-rater reliability and validity in patients in Korea.

\section{METHODS}

\section{Subjects}

After approval from the Institutional Review Board of the Catholic Medical Center of the Catholic University of Korea and a full explanation of the procedure, all participants and their guardians signed an informed consent form indicating that they agreed to participate in the study.

From 2008 to 2010, subjects were recruited from existing clinical populations visiting Saint Vincent Hospital, a secondary care center with an independent psychiatric unit of 36 beds in Suwon, Kunggi Province, Korea. A total of 156 subjects were recruited in this study including 59 patients with Alzheimer's disease $(\mathrm{AD})$ and depression and $62 \mathrm{AD}$ patients without depression. Two trained psychiatrists examined all of the subjects using the Provisional Diagnostic Criteria for Depression of Alzheimer Disease (PDCDA) by Olin et al. ${ }^{1}$ The inclusion criteria for the AD subjects were as follows: 1) age more than 65 years; 2) the National Institute of Neurological and Communication Disorders and Stroke/Alzheimer Disease and Related Disorders Association (NINCDS-ADRDA) diagnosis of probable $\mathrm{AD}^{13}$; and 3) Clinical Dementia Rating (CDR) of more than $1 .^{14}$ The exclusion criteria for the $\mathrm{AD}$ subjects were as follows: 1) a presumptive diagnosis of vascular dementia, dementia with Lewy body or other neurological or medical conditions that diminish cognitive function (e.g., hypothyroidism); or 2) a history of other psychiatric disorders attributable to psychotic symptoms (e.g., schizophrenia, delusional disorder and substance abuse).

Thirty-six age- and sex-matched healthy elderly controls (NC) were recruited from individuals visiting the hospital for regular health screening. All of the control subjects were also interviewed by trained psychiatrists and were ascertained to be free of major psychiatric problems.

\section{Procedures and assessment instruments}

\section{Korean Version of the Cornell Scale for Depression in Dementia (CSDD-K)}

The comparability of CSDD-K and the original CSDD was validated by stringent back-translation procedures. The CSDD was first translated into Korean by 2 psychiatrists and 1 psychologist, and any English phrases that were difficult to understand were translated into Korean after consulting a Korean professor of English literature. Then, the Korean CSDD was backtranslated by a person bilingual in English and Korean to validate the translation, and the back-translated version was reviewed. The content of the final CSDD-K was further verified by the back-translation procedure until the meaning of each item matched that of the original item in the CSDD. After obtaining permission from the original author of the CSDD, we established the final CSDD-K.

The CSDD-K is composed of 19 items that measure moodrelated signs, behavioral disturbances, physical signs, cyclic functions and ideational disturbances. All items of the CSDD-K are in Korean. Each item is measured on a 3-point continuum of 'absent (0)', 'mild or intermittent (1)', and 'severe (2)'. The maximum score is 38 , which would indicate the worst depressive symptom state in dementia patients.

\section{7-item Hamilton Depression Rating Scale (HAM-D ${ }_{17}$ )}

The Hamilton Depression Rating Scale (HAM-D) is an inventory of questions that is employed to detect and identify the intensity or severity of the signs and symptoms of depression in patients who have been diagnosed with clinical depression. ${ }^{15}$

The 17-item version of the HAM-D is more commonly used than the 21-item version, which contains four additional items that measure symptoms related to depression, such as paranoia and obsession, rather than the severity of the depressive symptoms themselves.

\section{5-item Korean Version of Geriatric Depression Scale $\left(\mathrm{GDS}_{15}\right)$}

The Geriatric Depression Scale (GDS) is one of the most widely used instruments for screening elderly depression. ${ }^{16}$ This scale involves 30-item self reporting inventories. To simplify this screening device for depression, Sheikh and Yesavage developed a 15-item GDS version that was proved to be an adequate substitute for the original GDS. ${ }^{17}$ Korean versions of GDS and $\mathrm{GDS}_{15}$ were validated by Bae et al.; the cut-off scores for depression in these tests were 10 and 8 , respectively. ${ }^{18}$

\section{Procedures}

The HAM- $\mathrm{D}_{17}$ was selected to prove the concurrent validity of the CSDD-K, which indicated that the results obtained us- 
ing this instrument were strongly correlated with measurements of a similar trait.

The raters in this study were seven experienced, board-certified psychiatrists who participated in formal consensus meetings concerning the use of the PDCDA, HAM-D ${ }_{17}$ and CSDD$K$. In the consensus meetings, an experienced supervisory psychiatrist and an actual test administrator observed the administration of the evaluations to two standard $\mathrm{AD}$ patients with depression via videotape recordings. All three meetings were held during the study period and the average attendance rate was $90.5 \%$. The inter-rater reliabilities of the PDCDA, HAM-D ${ }_{17}$ and CSDD-K were high with intra-class correlation coefficients (ICC) of $0.80,0.81$, and 0.82 , respectively. All of the raters of the PDCDA, HAM-D ${ }_{17}$ and CSDD-K were blinded during the test administration.

\section{Statistical analysis}

Group comparisons were performed using independent $\mathrm{t}$ tests and $\chi^{2}$ analyses to compare the quantitative and categorical variables, respectively. Internal consistency was assessed using Cronbach's $\alpha$. Test-retest reliability was estimated by calculating ICC. The concurrent validity between the CSDD-K and HAM-D $\mathrm{D}_{17}$ was evaluated using Pearson correlation coefficients. The factorial validity of the CSDD-K was examined using a principal components factor analysis with varimax rotation. The sensitivity, specificity and predictive values were calculated according to the standard formulae. We analyzed a receiver operating characteristic (ROC) curve to obtain the optimal cut-off score for detecting depression in dementia in $\mathrm{AD}$ subjects. In addition, pairwise comparison of ROC curves of CSDD-K, $\mathrm{HAMD}_{17}$ and $\mathrm{GDS}_{15}$ was conducted according to the methods of DeLong et al. ${ }^{19}$ Statistical analyses were conducted using SPSS for Windows, version 12.0, and Medcalc, version 12.1.4.

\section{RESULTS}

\section{Description of the sample}

Table 1 shows the baseline demographic data in our subject groups. The mean ages of the subjects in the $\mathrm{AD}$ with depression group, the $\mathrm{AD}$ without depression group and the $\mathrm{NC}$ group were $74.9(\mathrm{SD}=15.7), 75.1(\mathrm{SD}=14.6)$ and $74.2(\mathrm{SD}=14.3)$ years, respectively. The numbers of males in the $\mathrm{AD}$ with depression, AD without depression and NC groups were 25 (42.3\%), 26 (41.9\%) and 15 (41.6\%), respectively. No significant differences were found in age $(\mathrm{F}=1.303, \mathrm{p}=0.274)$ or gender ratio $\left(\chi^{2}=5.677, p=0.059\right)$ among the three groups. There was also no significant difference in education years among the three groups. CDR and MMSE-K scores were significantly different between $\mathrm{AD}$ and NC groups; however, no difference was observed between the $\mathrm{AD}$ with depression group and the $\mathrm{AD}$ without depression group.

\section{Reliability}

In the reliability test, Cronbach's a coefficient was 0.92 , which provided evidence of the internal consistency of the measure.

The test-retest reliability was examined by comparing the baseline CSDD-K score with that of a CSDD-K assessment performed 2 weeks later. The AD patients with depression only included those who claimed that there was no change in their depressive symptoms and who agreed to a second CSDD-K assessment. Forty-one of the $59 \mathrm{AD}$ patients with depression were recruited for the evaluation of the test-retest reliability. The test-retest reliability was $0.91(\mathrm{p}<0.0001)$.

\section{Validity}

Comparisons of different diagnostic groups

The mean CSDD-K scores of the $\mathrm{AD}$ with depression group,

Table 1. Demographic and clinical characteristics of study participants

\begin{tabular}{lccc}
\hline & AD with depression $(\mathrm{N}=59)$ & AD without depression $(\mathrm{N}=62)$ & $\mathrm{NC}(\mathrm{N}=36)$ \\
\hline Age $($ years $\pm \mathrm{SD})$ & $74.9 \pm 15.7$ & $75.1 \pm 14.6$ & $74.2 \pm 14.3$ \\
Education (years $\pm \mathrm{SD})$ & $9.6 \pm 2.7$ & $9.8 \pm 3.1$ & $10.1 \pm 2.4$ \\
Sex $(\mathrm{M}: \mathrm{F})$ & $25: 34$ & $26: 36$ & $15: 21$ \\
$\mathrm{CDR}(\text { mean } \pm \mathrm{SD})^{*}$ & $1.5 \pm 0.5$ & $1.4 \pm 0.3$ & 0 \\
$\mathrm{MMSE}-\mathrm{K}(\mathrm{mean} \pm \mathrm{SD})^{*}$ & $16.7 \pm 4.8$ & $17.1 \pm 3.9$ & $28.3 \pm 1.7$ \\
$\mathrm{CSDD}-\mathrm{K}(\text { mean } \pm \mathrm{SD})^{\dagger}$ & $13.5 \pm 5.5$ & $2.8 \pm 2.1$ & $2.1 \pm 2.1$ \\
$\mathrm{HAMD}_{17}(\text { mean } \pm \mathrm{SD})^{\dagger}$ & $17.8 \pm 6.5$ & $3.8 \pm 2.1$ & $3.5 \pm 2.6$ \\
$\mathrm{GDS}_{15}(\text { mean } \pm \mathrm{SD})^{\dagger}$ & $8.4 \pm 3.7$ & $1.8 \pm 0.7$ & $1.5 \pm 0.6$ \\
\hline
\end{tabular}

*significantly different between $\mathrm{AD}$ and $\mathrm{NC}(\mathrm{p}<0.001)$; no difference between $\mathrm{AD}$ with and without depression, ${ }^{\dagger}$ significantly different between $\mathrm{AD}$ with and without depression/ $\mathrm{NC}(\mathrm{p}<0.001)$; no difference between $\mathrm{AD}$ without depression and NC. AD: Alzheimer's disease, NC: healthy elderly controls, SD: standard deviation, CDR: clinical dementia rating, MMSE-K: Korean version of Mini Mental Status Examination, CSDD-K: Korean version of Cornell Scale for Depression in Dementia, HAMD 17 : 17 -item Hamilton Depression Rating Scale, GDS ${ }_{15}$ : 15-item Geriatric Depression Scale 
$\mathrm{AD}$ without depression group and NC group were 13.5 \pm 5.5 (95\% CI 12.3-14.5), 2.8 \pm 2.1 (95\% CI 2.0-3.7), and 2.1 \pm 2.1 (CI 1.5-2.8), respectively. One-way ANOVA was conducted to evaluate the extent to which the CSDD-K differentiated among $\mathrm{AD}$ patients with depression, $\mathrm{AD}$ patients without depression, and the NC group. There were significant differences in CSDD-K scores among the three groups $(F=110.0, p<0.001)$. In addition, post hoc analysis revealed a significant difference of CSDD-K scores between $\mathrm{AD}$ patients with depression and $\mathrm{AD}$ patients without depression $(\mathrm{p}<0.001)$; however, no difference was observed between the $\mathrm{AD}$ without depression group and the NC group ( $\mathrm{p}=0.8)$.

\section{Concurrent validity}

The relationship between the CSDD-K and the other scales (HAM-D ${ }_{17}$ and GDS $_{15}$ ) was assessed using correlation coefficients. We found that CSDD-K scores were relatively highly correlated with HAM-D $\mathrm{D}_{17}(\mathrm{r}=0.92, \mathrm{p}<0.001)$ and $\mathrm{GDS}_{15}(\mathrm{r}=$ $0.75, \mathrm{p}<0.001)$, respectively.

\section{Factorial structure of the CSDD-K}

To assess the construct validity of the CSDD-K, a principal components analysis (PCA) was performed for all subjects with $\mathrm{AD}$ on the 19 items of the scale (Table 2). Four components had eigen values $>1.0$, and 4 factors were extracted. A PCA using a varimax rotation was then performed. Factor loadings $>0.40$ were considered significant. The solution, which explained $63.8 \%$ of the variance, generated 'general depression' (anxiety, sadness, agitation, multiple physical complaints, diurnal variations of mood, suicide, poor self-esteem and pessimism); 'apathetic symptoms' (lack of reactivity to pleasant events, retardation, loss of interest, lack of energy, and mood congruent delusion); 'appetite symptoms' (appetite loss and weight loss) and 'sleep disturbances' (early morning awakening, difficulty falling asleep and multiple awakenings during sleep).

\section{ROC analysis}

The sensitivity, specificity and predictive values were calculated for every possible CSDD-K cut-off score, as well as for

Table 2. Factor loadings in the rotated factor matrix of CSDD-K $(\mathrm{N}=121)$

\begin{tabular}{|c|c|c|c|c|}
\hline \multirow{2}{*}{ Item } & \multicolumn{4}{|c|}{ Factor } \\
\hline & 1 & 2 & 3 & 4 \\
\hline \multicolumn{5}{|l|}{ Factor 1: general depression } \\
\hline Anxiety & 0.65 & 0.26 & 0.36 & 0.17 \\
\hline Sadness & 0.79 & 0.09 & 0.20 & 0.26 \\
\hline Agitation & 0.68 & 0.37 & 0.17 & 0.03 \\
\hline Multiple physical complaints & 0.52 & 0.17 & 0.15 & 0.36 \\
\hline Diurnal variations of mood & 0.46 & 0.35 & 0.30 & -0.21 \\
\hline Suicidal ideation & 0.77 & 0.08 & 0.01 & 0.10 \\
\hline Poor self esteem & 0.79 & 0.06 & 0.01 & 0.07 \\
\hline Pessimism & 0.58 & 0.31 & 0.08 & 0.32 \\
\hline \multicolumn{5}{|l|}{ Factor 2: apathetic symptoms } \\
\hline Lack of reactivity & 0.14 & 0.77 & -0.03 & 0.26 \\
\hline Retardation & 0.16 & 0.83 & 0.10 & -0.02 \\
\hline Loss of interest & 0.12 & 0.82 & 0.08 & 0.27 \\
\hline Lack of energy & 0.21 & 0.61 & 0.01 & 0.46 \\
\hline Mood congruent delusion & 0.29 & 0.61 & 0.19 & -0.17 \\
\hline \multicolumn{5}{|l|}{ Factor 3: appetite symptoms } \\
\hline Appetite loss & 0.13 & 0.06 & 0.80 & 0.15 \\
\hline Weight loss & 0.12 & 0.13 & 0.73 & 0.14 \\
\hline \multicolumn{5}{|l|}{ Factor 4: sleep disturbances } \\
\hline Difficulty falling asleep & 0.20 & -0.08 & 0.27 & 0.73 \\
\hline Multiple awakening & 0.08 & 0.19 & -0.01 & 0.84 \\
\hline Early morning awakening & 0.13 & 0.08 & 0.18 & 0.78 \\
\hline
\end{tabular}

CSDD-K: Korean version of Cornell Scale for Depression in Dementia

Table 3. The cut-off scores, sensitivity, specificity, and predictive values resulting from receiver operation characteristics (ROC) analyses of the CSDD-K, HAMD ${ }_{17}$ and $\mathrm{GDS}_{15}$ in AD patients

\begin{tabular}{|c|c|c|c|c|c|c|}
\hline & AUC & Cut-off & Sensitivity (\%) & Specificity (\%) & PPV (\%) & NPV (\%) \\
\hline \multirow[t]{3}{*}{ CSDD-K } & 0.98 & 6 & 90.6 & 96.0 & 98.9 & 72.7 \\
\hline & & 7 & 87.5 & 100 & 100 & 69.6 \\
\hline & & 8 & 82.2 & 100 & 100 & 61.0 \\
\hline \multirow[t]{3}{*}{$\mathrm{HAMD}_{17}$} & 0.96 & 8 & 95.8 & 80.0 & 94.8 & 83.3 \\
\hline & & 9 & 94.8 & 88.0 & 96.8 & 81.5 \\
\hline & & 10 & 87.5 & 88.0 & 96.6 & 64.7 \\
\hline \multirow[t]{3}{*}{ GDS } & 0.80 & 4 & 81.2 & 64.0 & 89.7 & 47.1 \\
\hline & & 5 & 72.9 & 76.0 & 92.1 & 42.2 \\
\hline & & 6 & 64.5 & 80.0 & 92.5 & 37.0 \\
\hline
\end{tabular}

CSDD-K: Korean version of Cornell Scale for Depression in Dementia, HAMD 17 $_{17}$ 17-item Hamilton Depression Rating Scale, GDS ${ }_{15}$ : 15-item Geriatric Depression Scale, AUC: area under curve, PPV: positive predictive value, NPV: negative predictive value, AD: Alzheimer's disease 


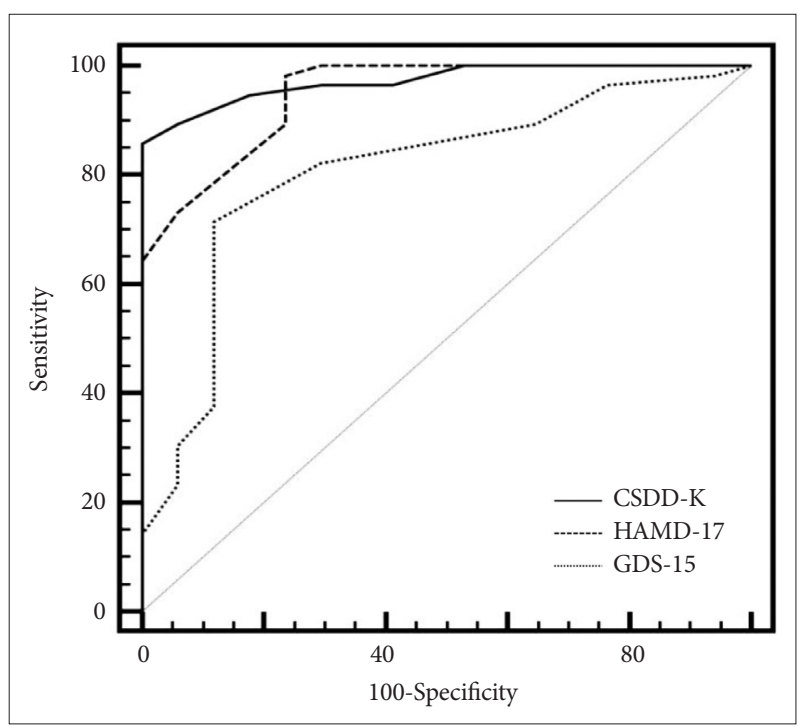

Figure 1. The ROC curves of CSDD-K, HAMD17 and GDS for the prediction of the diagnosis of depression in AD. ROC: Receiver Operation Characteristics, CSDD-K: Korean version of Cornell Scale for Depression in Dementia, HAMD 17 : 17-item Hamilton Depression Rating Scale, GDS ${ }_{15}$ : 15 -item Geriatric Depression Scale, AD: Alzheimer's disease.

those of $\mathrm{HAMD}_{17}$ and $\mathrm{GDS}_{15}$. Table 3 shows three different threshold scores and the corresponding sensitivity, specificity, and positive and negative predictive values of the CSDD-K, $\mathrm{HAMD}_{17}$ and $\mathrm{GDS}_{15}$. A cut-off score of 7 yielded a sensitivity of $87.5 \%$ and specificity of $100 \%$. On the other hand, the cutoff scores were a total score of 9 in $\mathrm{HAMD}_{17}$ (sensitivity $94.8 \%$ and specificity $88.0 \%$ ) and 5 in GDS $_{15}$ (sensitivity $72.9 \%$ and specificity $76.0 \%)$.

The area under curve (AUC) of CSDD-K, HAMD ${ }_{17}$ and $\mathrm{GDS}_{15}$ were $0.98,0.96$ and 0.80 , respectively (Figure 1 ). There were significant differences in AUC between CSDD-K and $\mathrm{GDS}_{15}(\mathrm{p}=0.0076)$ and between HAMD $\mathrm{H}_{17}$ and $\mathrm{GDS}_{15}(\mathrm{p}=$ $0.0140)$, respectively. However, no significant difference was observed in AUC between CSDD-K and $\mathrm{HAMD}_{17}(\mathrm{p}=0.440)$.

\section{DISCUSSION}

The present study was performed in order to construct a Korean version of the CSDD and to provide data concerning its psychometric properties, construct validity, and factor structure.

In previous validation studies of the CSDD from other countries, Cronbach's a was between 0.79 and 0.93 and the testretest reliability coefficient was within $0.73-0.99 .^{8-11,20,21}$ In the present study, Cronbach's a was 0.92 and the test-retest reliability coefficient was 0.91 . Thus, the CSDD-K scores for the $\mathrm{AD}$ and $\mathrm{NC}$ groups obtained in the present study were similar to those reported in previous studies conducted in other countries.
In the present study, $\mathrm{HAMD}_{17}$ and $\mathrm{GDS}_{15}$ were conducted in order to evaluate the convergent validity of the CSDD-K. The CSDD-K was more strongly correlated with HAM- $\mathrm{D}_{17}$ $(\mathrm{r}=0.92, \mathrm{p}<0.001)$ than with $\mathrm{GDS}_{15}(\mathrm{r}=0.75, \mathrm{p}<0.001)$, which was in line with previous studies. ${ }^{9,12}$ This might be due to the similar content and interview processes (clinician rating scales) of HAM-D $\mathrm{D}_{17}$ and CSDD-K in the evaluation of depressive symptoms.

Our results suggested that a four-factor solution explaining $63.8 \%$ of the variance was acceptable for the CSDD-K. This result was in accordance with other factor analyses reporting 4- or 5-factor solutions. A previous factor analysis by Harwood et al. reported 4-factor solutions (general depression, rhythm disturbances, agitation/psychosis and negative symptoms) accounting for $43.1 \%$ of common variance. ${ }^{22}$ Another factor analysis on Chinese dementia patients resulted in 5-factor solutions (depression, cyclic functions, physical signs, behavioral disturbances, mood signs) explaining $61.2 \%$ of variance. ${ }^{9} \mathrm{Al}$ though the original study of Alexopoulos et al. suggested five clusters of mood-related signs, behavioral disturbance, physical signs, cyclic functions and ideational disturbance, the previous factor analyses yielded various factor solutions of subitems of the CSDD. ${ }^{7,922,23}$ These discrepancies might be due to the differences in the clinical settings or diagnosis and inhomogeneity of depressive symptoms in the subjects.

We conducted ROC analysis to obtain the CSDD-K cut-off score for the diagnosis of depression in $\mathrm{AD}$. In this study, a cut-off value of 7 yielded a sensitivity of $87.6 \%$ and specificity of $100 \%$. Validity studies have reported different results of its power to detect a depressive disorder and various cut-off points to this end have been recommended. As they did not conduct ROC analysis, Alexopoulos et al. ${ }^{7}$ did not recommend any cut-off point in their original study. Later on, a cut-off point of 8 was regarded as suitable for a minor depressive episode. Some studies showed lower cut-off points for depression, between 4 and 6, while others reported higher cut-off points, between 9 and 12. ${ }^{12,21,24,25}$ This variability of cut-off points for a depressive disorder in dementia might be due to methodological differences between the studies.

In addition to the ROC analysis of CSDD-K, ROC curves of $\mathrm{HAMD}_{17}$ and $\mathrm{GDS}_{15}$ were also obtained. The area under the curve (AUC) is an overall index of the accuracy of the discrimination provided by CSDD-K, $\mathrm{HAMD}_{17}$ and $\mathrm{GDS}_{15}$. An AUC of 0.5 implies random performance, whereas an AUC of 1.0 represents perfect performance. Generally, an AUC above 0.85 is considered to be an indication of good diagnostic capability. The AUC of CSDD-K, HAMD ${ }_{17}$ and GDS $_{15}$ were 0.98, 0.96 and 0.80 , respectively. These results were in line with previous studies. Mayer et al. ${ }^{26}$ suggested that CSDD was more sensitive than HAMD in the detection of treatment effects at all 
levels of depression severity. Another study by Kørner et al. ${ }^{12}$ showed that CSDD had higher sensitivity and specificity than $\mathrm{GDS}_{15}$ in diagnosing depression in dementia. In this study, the AUCs of CSDD-K and $\mathrm{HAMD}_{17}$ were not significantly different. This might be attributable to the subject characteristics of our study. Most of the AD subjects had milder degree dementia (mean $\mathrm{CDR}=1.5 \pm 0.5$ ) and were recruited from an outpatient clinic. As the original CSDD was developed for application in nursing home settings, the discrimination power enhanced by interviewing patients' caregivers would be reduced in patients with milder degree dementia.

The findings of this study have to be cautiously interpreted due to the following limitations. First, as we recruited AD patients with or without depression who visited the outpatient clinic in this study, our results should not be generalized to other types of dementias or to other clinical settings such as nursing homes and inpatient treatment programs. Further validation trials including various types of dementias and patients in various clinical settings will be needed. Second, we used the PDCDA criteria as the gold standard for diagnosing depression in dementia. A previous study showed the cut-off score of the CSDD changed according to the different gold standards, such as DSM-IV-TR, ICD-10 or PDCDA. ${ }^{27}$ Thus, comparison of the cut-off scores of the CSDD-K according to various gold standards will be needed.

\section{Conclusions}

Our data demonstrate that the CSDD-K is a reliable measure with good psychometric properties and can be used as a reliable, valid, and timesaving tool to diagnose and assess depression in dementia. The data collected in the present study may serve as a baseline for comparison with clinical samples in future studies in the Korean population.

\section{Acknowledgments}

This research was supported by Basic Science Research Program through the National Research Foundation of Korea (NRF) funded by the Ministry of Education, Science and Technology (2012R1A1A2042339).

\section{REFERENCES}

1. Olin JT, Katz IR, Meyers BS, Schneider LS, Lebowitz BD. Provisional diagnostic criteria for depression of Alzheimer disease: rationale and background. Am J Geriatr Psychiatry 2002;10:129-141.

2. Steele C, Rovner B, Chase GA, Folstein M. Psychiatric symptoms and nursing home placement of patients with Alzheimer's disease. Am J Psychiatry 1990;147:1049-1051.

3. Gonzlez-Salvador T, Lyketsos CG, Baker A, Hovanec L, Roques C, Brandt J, et al. Quality of life in dementia patients in long-term care. Int J Geriatr Psychiatry 2000;15:181-189.

4. Tariot PN, Mack JL, Patterson MB, Edland SD, Weiner MF, Fillenbaum $\mathrm{G}$, et al. The behavior rating scale for dementia of the consortium to establish a registry for Alzheimer's disease. The behavioral pathology committee of the consortium to establish a registry for Alzheimer's disease. Am J Psychiatry 1995;152:1349-1357.
5. Cummings JL. The Neuropsychiatric Inventory: assessing psychopathology in dementia patients. Neurology 1997;48(5 Suppl 6):S10-S16.

6. Lyketsos CG, Lee HB. Diagnosis and treatment of depression in Alzheimer's disease. A practical update for the clinician. Dement Geriatr Cogn Disord 2004;17:55-64.

7. Alexopoulos GS, Abrams RC, Young RC, Shamoian CA. Cornell Scale for Depression in Dementia. Biol Psychiatry 1988;23:271-284.

8. Amuk T, Karadag F, Oguzhanoglu N, Oguzhanoglu A. Reliability and validity of the Cornell Scale for Depression in Dementia in an elderly Turkish population. Turk Psikiyatri Derg 2003;14:263-271.

9. Lin JN, Wang JJ. Psychometric evaluation of the Chinese version of the Cornell Scale for Depression in Dementia. J Nurs Res 2008;16:202-210.

10. Schreiner AS, Hayakawa H, Morimoto T, Kakuma T. Screening for late life depression: cut-off scores for the Geriatric Depression Scale and the Cornell Scale for Depression in Dementia among Japanese subjects. Int J Geriatr Psychiatry 2003;18:498-505.

11. Carthery Goulart MT, Areza Fegyveres R, Schultz R, Okamoto I, Caramelli P, Bertolucci PH, et al. Brazilian version of the Cornell depression scale in dementia. Arq Neuropsiquiatr 2007;65:912-915.

12. Kørner A, Lauritzen L, Abelskov K, Gulmann N, Marie Brodersen A, Wedervang-Jensen T, et al. The Geriatric Depression Scale and the Cornell Scale for Depression in Dementia. A validity study. Nord J Psychiatry 2006;60:360-364.

13. McKhann G, Drachman D, Folstein M, Katzman R, Price D, Stadlan EM. Clinical diagnosis of Alzheimer's disease: report of the NINCDSADRDA Work Group under the auspices of Department of Health and Human Services Task Force on Alzheimer's Disease. Neurology 1984;34:939-944.

14. Morris JC. The Clinical Dementia Rating (CDR): current version and scoring rules. Neurology 1993;43:2412-2414.

15. Hamilton M. Development of a rating scale for primary depressive illness. Br J Soc Clin Psychol 1967;6:278-296.

16. Yesavage JA, Brink TL, Rose TL, Lum O, Huang V, Adey M, et al. Development and validation of a geriatric depression screening scale: a preliminary report. J Psychiatr Res 1982;17:37-49.

17. Sheikh VI, Yesavage VA. Geriatric Depression Scale (GDS): Recent Evidence and Development of Shorter Version. New York: Haworth Press; 1986.

18. Bae JN, Cho MJ. Development of the Korean version of the Geriatric Depression Scale and its short form among elderly psychiatric patients. J Psychosom Res 2004;57:297-305.

19. DeLong ER, DeLong DM, Clarke-Pearson DL. Comparing the areas under two or more correlated receiver operating characteristic curves: a nonparametric approach. Biometrics 1988;44:837-845.

20. Camus V, Schmitt L, Ousset PJ, Micas M. Depression and dementia: contribution to the French validation of 2 depression scales: the Cornell Scale for Depression in Dementia and the Dementia Mood Assessment Scale. Encephale 1995;21:201-208.

21. Lam CK, Lim PP, Low BL, Ng LL, Chiam PC, Sahadevan S. Depression in dementia: a comparative and validation study of four brief scales in the elderly Chinese. Int J Geriatr Psychiatry 2004;19:422-428.

22. Harwood DG, Ownby RL, Barker WW, Duara R. The factor structure of the Cornell Scale for Depression in Dementia among probable Alzheimer's disease patients. Am J Geriatr Psychiatry 1998;6:212-220.

23. Schreiner AS, Morimoto T. Factor structure of the Cornell Scale for Depression in Dementia among Japanese poststroke patients. Int J Geriatr Psychiatry 2002;17:715-722.

24. Vida S, Des Rosiers P, Carrier L, Gauthier S. Depression in Alzheimer's disease: receiver operating characteristic analysis of the Cornell Scale for Depression in Dementia and the Hamilton Depression Scale. J Geriatr Psychiatry Neurol 1994;7:159-162.

25. Logsdon RG, Teri L. Depression in Alzheimer's disease patients: caregivers as surrogate reporters. J Am Geriatr Soc 1995;43:150-155.

26. Mayer LS, Bay RC, Politis A, Steinberg M, Steele C, Baker AS, et al. Comparison of three rating scales as outcome measures for treatment trials 
Reliability and Validity of the CSDD-K

of depression in Alzheimer disease: findings from DIADS. Int J Geriatr Psychiatry 2006;21:930-936.

27. Barca ML, Engedal K, Selbaek G. A reliability and validity study of the cornell scale among elderly inpatients, using various clinical criteria. Dement Geriatr Cogn Disord 2010;29:438-447. 